\title{
Information Extraction in a Set of Knowledge Using a Fuzzy Logic Based Intelligent Agent
}

\author{
Jorge Ropero, Ariel Gómez, Carlos León, and Alejandro Carrasco \\ Department of Electronic Technology, University of Seville, Spain \\ jropero@dte.us.es, ariel@us.es, cleon@us.es, acarrasco@us.es
}

\begin{abstract}
A method for Information Extraction (IE) in a set of knowledge is proposed in this paper in order to answer to user consultations using natural language. The system is based on a fuzzy logic engine, which takes advantage of its flexibility for managing sets of accumulated knowledge. These sets can be built in hierarchic levels by a tree structure. A method of consultation based on a fuzzy logic application provided with an interface that one may interact with in natural language is also proposed. The eventual aim of this system is the implementation of an intelligent agent to manage the information contained in an internet portal.
\end{abstract}

Keywords: Fuzzy Logic, Intelligent Agent, Information Extraction, Vector Space Model.

\section{Motivations}

The approach to the contents of an extensive set of accumulated knowledge -a database, a summary of documents, web contents, goods in a store, pictures, etc - is an important concern nowadays. Information Retrieval (IR) deals with large collections of textual material, and its aim is to satisfy user queries and needs [1]. These needs meet increased when the matter is not in the form of text, the user in question is not a habitual of the matter, there are ambiguous contents, bad organization or, simply, complex topics or a great amount of information difficult to manage.

Eventually, unsuccessful attempts can turn out to be frustrating if the exact term or terms are not used to make the consultations - a machine only will answer adequately if it is asked in an exact way -, and one can eventually end in a paradox: the less one knows the more difficult it is to find the answers. In many cases the solution is to seek help from an expert on the topic. In fact the person asked to help is an interpreter who is able to generate a syntactically and semantically a correct search obtaining the desired answers. Consequently, there is the need for an agent to interpret the vague information we provide, giving us concrete answers related to the existing contents of the set of knowledge. This should be based on an estimation of the certainty of the relation between what we have expressed in natural language and the contents stored in the set of knowledge. To solve this, this paper proposes a method to extract information from a webportal. This method is founded on a Vector Space Model (VSM) based classification 
of contents by creating a few indexes based on key words, and a method of consultation based on a Fuzzy Logic (FL) application with an interface that one may interact with in natural language. We then propose an artificial intelligence (AI) application based on the use of FL.

\section{Related Work}

As said above, the quantity of web information grows exponentially. For a long time researchers have faced the problem of managing this high amount of information IR research deals predominantly with documents. Achieving both high recall and precision in IR is one of its most important objectives. IR has been widely used for text classification [2, 3] introducing approaches such as Vector Space Model (VSM), $\mathrm{K}$ nearest neighbour method (KNN), Bayesian classification model, neural networks and Support Vector Machine (SVM) [4]. VSM is the most frequently used model.

Moreover, the use of unlabeled, distributed and heterogeneous web data has prompted the need for developing automatic mining techniques on the World Wide Web, giving rise to the term web mining [5]. Many techniques like Web Content Mining (WCM), Web Structure Mining (WSM) or Web Usage Mining (WUM) have been used in the last years [6]. WCM and IR are separated by a thin line [5]: they are both document finding oriented. The main difference between them lies on the use of semistructured data on the web. This makes WCM especially challenging [7]. WSM deals with web structure itself. WSM techniques enable improving web contents or extracting knowledge and other required information. The major methods of Information Extraction (IE) involve writing wrappers [5]. Some examples of the use of wrappers for IE are STAVIES, which presents a fully automated IE method for Web pages [8] or OMINI [9].

What we propose is a fully novel method using Fuzzy Logic (FL) not only to extract the required information but making the most of FL flexibility to extract related information too, giving answers to user consultations in natural language and bearing in mind that non-expert users tend not to be exact in their searches.

\section{Mode of Operation}

\subsection{Objectives}

The main objective of the system designed must be to let the users find possible answers to what they are looking for in a huge set of knowledge. With this aim, the whole set of knowledge must be classified into different objects, as shown in Figure 1. These objects are the possible user consultations, organized in hierarchic groups. A standard question is assigned for every object, and different key words from each standard question must then be selected in order to differentiate one object from the others. Finally, term weights are assigned to every word for every level of hierarchy in a scheme based on a vector space model. These term weights are the inputs to a fuzzy logic system, which must detect the object to which the correspondent user consultation refers. Term weighting scheme is shown in Figure 2. 


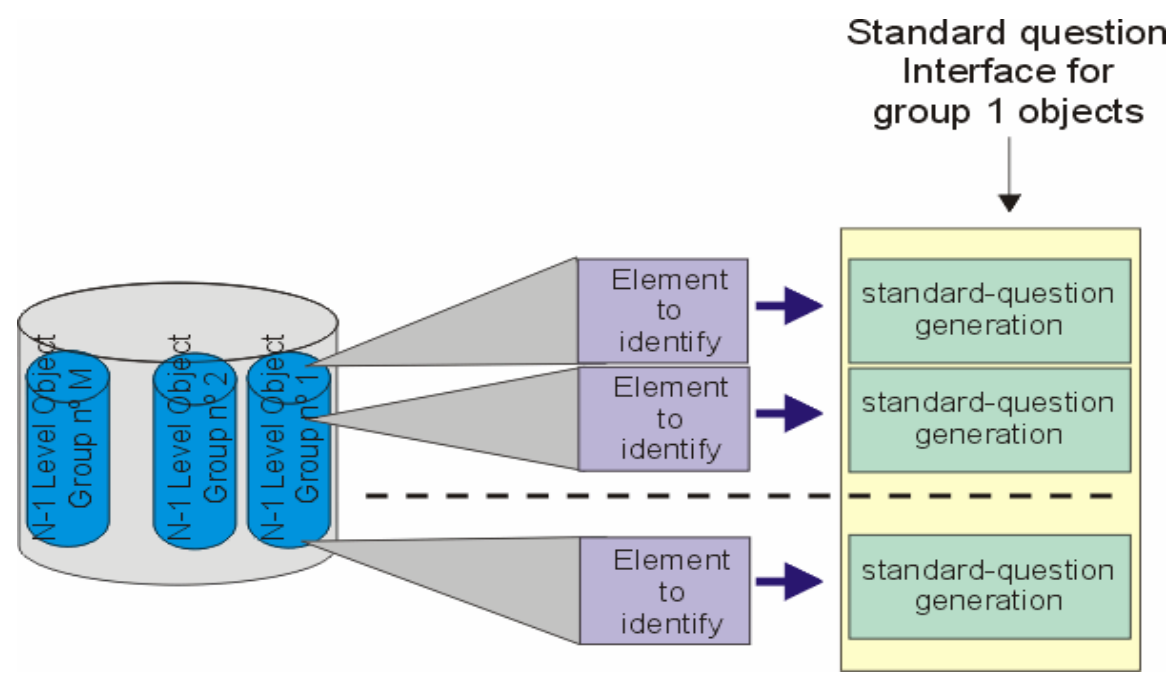

Fig. 1. Generated standard question interface

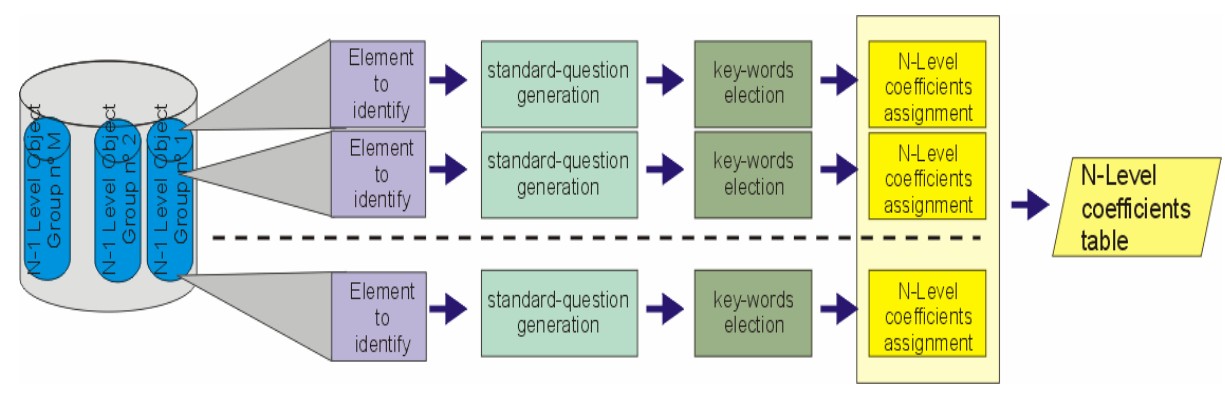

Fig. 2. Term weighting scheme

\subsection{Hierarchic Structure}

The standard-questions collection generated from the whole set of knowledge must be organized in hierarchic groups. This offers the advantage of easier handling. For tests, we decided to use the questions-answers database of the University of Seville to create a list of the most frequently asked questions. The whole knowledge was organized into three levels: topic, section and question. For any web page, it would be enough to create a bank of possible questions-answers, arrange them hierarchically, and identify them as objects.

Every possible user question must be related with standard questions in order to present its standard answers as possible answers to this user question. This may be seen in Figure 3. There are obviously many ways of asking, so the aim of the system is to identify real user consultations and what we have called standard questions. As there may be several questions similar to the one asked by the user or as it may be interesting for the user to get related answers, several answers will be presented. 


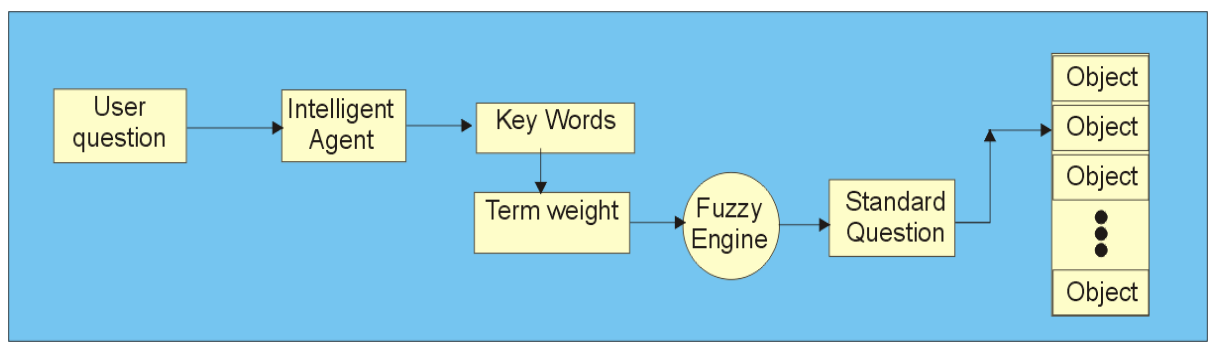

Fig. 3. Mode of operation

When a question is made, the first processing step consists of distinguishing the key words in the consultation. These words must be searched in a database which must contain all the words that are related somehow to the content of the subject we are dealing with -see Figure 4. Another database with the possible answers becomes necessary.

\begin{tabular}{|c|c|c|c|c|c|c|}
\hline \multicolumn{4}{|c|}{ 击 Tema1Nivel2Apdo2 : Tabla } & \multirow{2}{*}{\multicolumn{2}{|c|}{ - $\square$}} & $x$ \\
\hline & Campo1 & Campo2 & Campo3 & & & $\Delta$ \\
\hline \multirow[t]{3}{*}{ 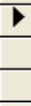 } & colegiados & 0,8 & 0 & & 0 & \\
\hline & equipo & 0 & 0,5 & & 0 & \\
\hline & gobierno & 0 & 0,8 & & 0 & \\
\hline & organos & 0,9 & 0 & & 0 & \\
\hline & puestos & 0 & 0 & & 0,7 & \\
\hline & relacion & 0 & 0 & & 0,7 & \\
\hline & sevilla & 0,2 & 0,2 & & 0,2 & \\
\hline & trabajo & 0 & 0 & & 0,7 & \\
\hline & universidad & 0,3 & 0,3 & I & 0,3 & - \\
\hline & gistro: $\mathbf{1 4} \mid \mathbf{4}$ & $\bullet 1$ & 米 de 9 & & & L \\
\hline
\end{tabular}

Fig. 4. Index words database

Key words are assigned to every standard question in order to identify it. These words are chosen among those that could appear in a possible consultation. As mentioned above, the whole knowledge is grouped in various hierarchic levels, so the belonging of these words to different levels is determined by a few numerical coefficients indicating how significant the considered word is within the level in question. With this aim, weight vectors are assigned to each word. Each vector contains the certainty of belonging to every fuzzy set. It is important to notice that the same word can belong to several different sets.

\subsection{Term Weighting}

As the system must work using a natural language interface, we propose the use of a system based on VSM for term weighting. Automatic document indexing is usually based on the frequency of occurrence, that is to say, words with a high frequency of occurrence are less significant than words with a low one, and the inverse document 
frequency, that is, how often it occurs in the whole document. The main limitation of this scheme is created by terms which are unhelpful for identification but have the same frequency of occurrence as the relevant ones [3,4,10-14].

Thus, we propose a novel scheme based on a vector space model which also takes into account if the word is important for the meaning of the question itself or if the word is linked to other words and its relationship with the whole set of knowledge for term weighting $[3,15]$.

The success of the proposed method depends to a great extent on a correct assignment of the coefficients to the key words, that is, the building of the weight vectors. The process consists of 3 stages: election of key words, coefficient assignment of the chosen words; and modification of the index values in order to obtain the desired minimal certainties.

Election of key words. As mentioned earlier, for every element that has to be determined, a question is assigned in natural language. These questions are called standard questions. From every standard question key words are chosen. These key words will allow the user consultation and these standard questions to be matched and, therefore, to indicate the required element. Word election is based on its concretion, meaning the degree of relation of the word with the element to be identified. This excludes articles, conjunctions, verb forms, etc, unless they are strongly significant for the question structure.

Coefficient assignment. For every key word, coefficients corresponding to every level of hierarchy of the whole set of knowledge must be assigned. The higher the relationship between the present level and the key word, the higher the coefficient of that key word will be for that level. These coefficients are the inputs to every fuzzy logic system, as described in Section 2.4.

Standard question recognition test. Once the coefficient assignment is made, standard question recognition tests must take place. These tests are based on using standard questions as user consultations.

Coefficient modification. The results obtained for standard question recognition tests represent the relationship of every standard-question relationship with itself. The higher it is the better the results are. However, it must be taken into account that similar questions must have a high degree of certainty too. This allows for the modification of coefficients in order to achieve better results. An example of how we have modified some coefficients is shown in Section 3.1.

\subsection{Fuzzy Logic System}

Fuzzy logic arises as a response to the inflexibility of the classic binary logic [16-17]. By means of a set of functions, a degree of flexibility may be given to these epithets: what may be cool for a Sevillian might be mild for a Berliner.

A fuzzy logic system gives flexibility for term weighting. More important than having a concrete value for weights, what really matters is that a feature is represented by a word. It is not so important that a weight is 0.8 or 0.9 : the weight is HIGH in both cases. 
All the key words are extracted for comparison with the ones contained in our key word database. As mentioned earlier, the whole set of knowledge is arranged in levels of hierarchy. The inputs to the fuzzy logic system are the coefficients of belonging to every level. The set "belonging to every level 1" is analyzed bearing in mind the value returned by the fuzzy engine. If the level of certainty is lower than a predefined value, the content of the corresponding set is rejected. Starting from level one and using a tree structure makes it possible to reject a great amount of content which will not be considered in future searches.

For every set that has overcome a minimum certainty threshold, the process is repeated and the coefficients of belonging corresponding to every level 2 set are evaluated. Sets where the degree of certainty returned by the fuzzy engine does not surpass a certain minimal threshold are rejected. If they surpass the threshold, the method for determining the belonging to the following level is applied to them. This process is repeated until the last level. The answers correspond to those last level elements in which certainty has overcome the definite threshold. There can be more than one answer. The vaguer the questions, the more answers we will obtain. This process may be seen in Figure 5.

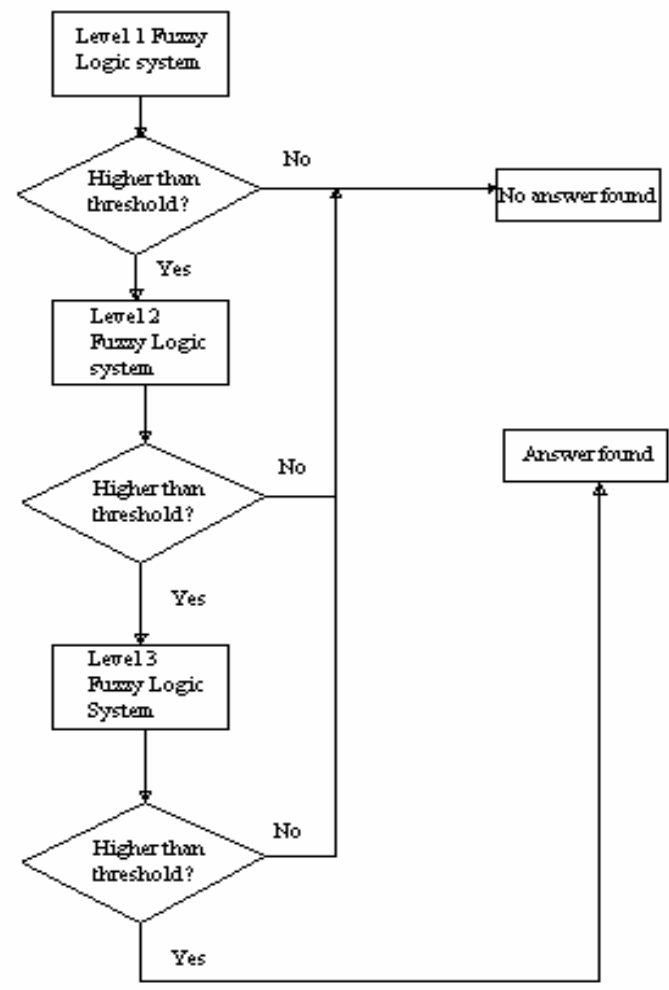

Fig. 5. Fuzzy logic system: operation mode 
The heart of the fuzzy logic system is the fuzzy engine. This engine is responsible for determining the probability that the key words contained in a consultation will belong to a certain fuzzy set in a specific level. The engine must evaluate the belonging to every set for the corresponding level. Thus, the engine takes the coefficients of the key words for that set as inputs. The fuzzy engine output will be determined by the defined rules. These rules are of the IF ... THEN type. An example of a rule might be this:

IF word_index 1 is HIGH AND word_index 2 is MEDIUM AND word_index 3 is LOW, THEN output is HIGH.

\subsection{System Administrator}

The system requires a system administrator who has basically three functions:

a) Defining and modifying term weights and rules.

b) Adding new words to the database when necessary.

c) Creating a system feedback which asks the eventual users for their opinion about the answers given by the assistant in order to take the necessary steps in each case.

Thus far, an administrator interface has been created - Figure 6 - .

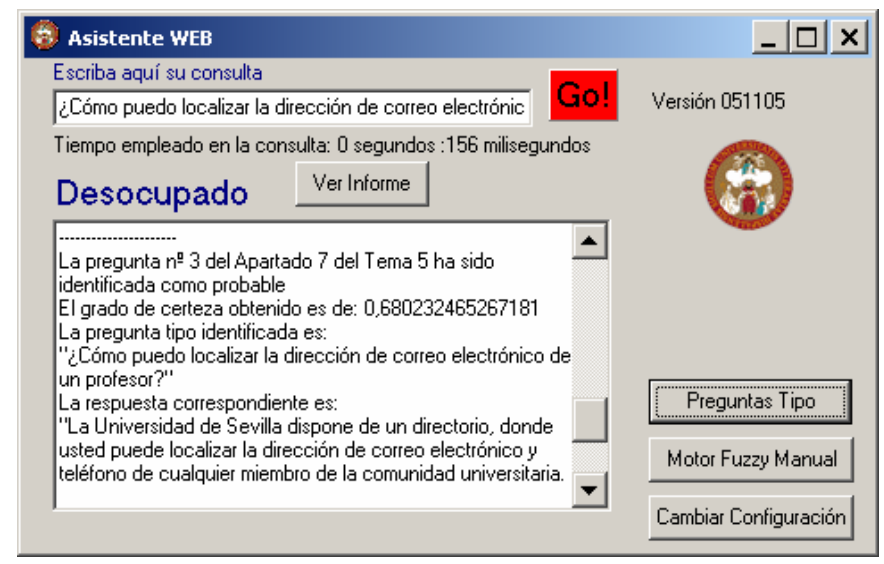

Fig. 6. Administrator interface

\section{Tests and Results}

\subsection{Test Definition}

Tests are based on the use of standard questions as user consultations. The first goal of these tests is to check that the system makes a correct identification of standard questions with an index of certainty higher than 0.7 . The use of fuzzy logic makes it possible to identify not only the corresponding standard question but others as well. This is related to recall, though it does not match that exact definition [18]. The 
second goal is to check if the required standard question is among the three answers with higher degree of certainty. These three answers should be presented to the user. The correct answer must be among these three options. This is related to precision, though it does not match that exact definition either.

To achieve these goals, it will be necessary to modify the assigned coefficients in principle. These modifications will be carried out again using a bottom-up design, so that the certainty returned by the fuzzy engine is at least of 0.7 for the correct decision in the lowest level, and of 0.5 for other levels. Likewise, in order to limit the number of possible answers, incorrect question indexes may be modified making them lower.

This method has been proven considering the most frequent questions asked in the portal web administrator as our set of knowledge, which consists on a set of 117 questions. The elements to be found are the questions themselves and possibly related questions.

Test results for standard question recognition fit into five categories:

1.-The correct question is the only one found or the one that has the highest degree of certainty.

2.-The correct question is between the two with the highest certainty or is the one that has the second highest degree of certainty.

3.-The correct question is among the three with the highest degree of certainty or is the one that has the third highest certainty.

4.-The correct question is found but not among the three with the highest degree of certainty

5.-The correct question is not found.

\subsection{Rule Definition}

As mentioned above, rule definition corresponds to the administrator. Logically, the more inputs the engine has, the more rules there are. For example, for a three input engine, the inputs can take three values: LOW, MEDIUM and HIGH. The outputs can take the values of LOW, MEDIUM-LOW, MEDIUM-HIGH and HIGH. The inference rules defined are:

If all inputs are LOW, output is LOW.

If one input is MEDIUM and the others are LOW, output is MEDIUM-LOW.

If two inputs are MEDIUM and the others are LOW, output is MEDIUM-HIGH.

If all the input are MEDIUM or one input is HIGH, output is HIGH.

The possible combinations generate 27 rules for the fuzzy engine. A five input engine generates 243 rules.

\subsection{Input Definition}

For every question, 3 to 5 key words are defined. Nevertheless, the user may include in his consultation anywhere from 1 to 5 of these words. Defining an engine with only a few inputs causes rapid saturation of the system. This is a great handicap for precision: $90 \%$ of correct answers are detected but only half of them are the first 
option as may be seen in Table 1. Defining a five input engine produces values with a very low degree of certainty. Precision grows to $55 \%$ but recall falls.

A solution to this problem is the use of variable thresholds. When all eventual outputs are below the fixed threshold, this threshold goes down until an output is found. If thresholds are variable, the system is more flexible and results are better.

Finally, the solution provided is to implement a flexible system with variable inputs. If the user consultation has at the most three key words, a three input fuzzy engine is used, whereas if the user consultation includes four or more key words, the system will use a five input fuzzy engine. Results are much better in terms of recall and precision. The user obtains the correct answer $97.75 \%$ of the times and the first option $77.45 \%$ of the times.

Table 1. Test results

\begin{tabular}{lccccc}
\hline $\begin{array}{l}\text { Type of system / Test } \\
\text { result category }\end{array}$ & $\begin{array}{c}\text { First } \\
\text { answer }\end{array}$ & $\begin{array}{c}\text { Among the first } \\
\text { two answers }\end{array}$ & $\begin{array}{c}\text { Among the first } \\
\text { three answers }\end{array}$ & $\begin{array}{c}\text { Out of the first } \\
\text { three answers }\end{array}$ & $\begin{array}{c}\text { Failed } \\
\text { answer }\end{array}$ \\
\hline $\begin{array}{l}3 \text { input engine } \\
5 \text { input system with }\end{array}$ & $45 \%$ & $24 \%$ & $9 \%$ & $12 \%$ & $10 \%$ \\
$\begin{array}{l}\text { fixed thresholds } \\
5 \text { input system with }\end{array}$ & $55 \%$ & $12 \%$ & $3 \%$ & $1 \%$ & $29 \%$ \\
$\begin{array}{l}\text { variable thresholds } \\
\begin{array}{l}\text { Variable input system } \\
\text { with fixed thresholds }\end{array}\end{array}$ & $70 \%$ & $14 \%$ & $3 \%$ & $1 \%$ & $12 \%$ \\
\hline
\end{tabular}

\section{Conclusions}

A method of consultation based on a fuzzy logic application provided with an interface that one may interact with in natural language has been presented. The system takes advantage of converting any kind of object in a text object; this allows the application of text retrieval techniques. The other advantage of the method arises from fuzzy logic flexibility, which makes it possible to have a non-rigid term weighting in the stage of classification of the contents in the set of knowledge.

A method for IE in a set of knowledge is also proposed. We also present a modification of the classical VSM to define term weights, which are used as inputs to a fuzzy logic based system.

An eventual application of this system is the implementation of an intelligent agent to manage the information contained in an internet portal. So far the results obtained are good enough, as the number of correctly detected consultations is high.

\section{References}

1. Kwok, K.L.: A neural network for probabilistic information retrieval. In: Proceedings of the 12th annual international ACM SIGIR conference on Research and development in information retrieval. Cambridge, Massachusetts, United States (1989)

2. Aronson, A.R., Rindflesch, T.C., Browne, A.C.: Exploiting a large thesaurus for information retrieval. In: Proceedings of RIAO, pp. 197-216 (1994) 
3. Liu, S., Dong, M., Zhang, H., Li, R., Shi, Z.: An approach of multi-hierarchy text classification 2001. In: International Conferences on Info-tech and Info-net Proceedings. ICII 2001 - Beijing. vol. 3, Oct/ Nov. 2001 p. 95-100 (2001)

4. Lu, M., Hu, K., Wu, Y., Lu, Y., Zhou, L.: SECTCS: towards improving VSM and Naive Bayesian classifier. In: 2002 IEEE International Conference on Systems, Man and Cybernetics.vol. 5, p. 5 (October 2002)

5. Pal, S.K., Talwar, V., Mitra, P.: Web Mining in Soft Computing Framework: Relevance, State of the Art and Future Directions. In: IEEE Transactions on Neural Networks Vol. 13(5), pp 1163-1177 (September 2002)

6. Rios, S.A., Velásquez, J.D., Yasuda, H., Aoki, T.: Conceptual Classification to Improve a Web Site Content. In: Corchado, E., Yin, H., Botti, V., Fyfe, C. (eds.) IDEAL 2006. LNCS, vol. 4224, pp. 869-877. Springer, Heidelberg (2006)

7. Chakrabarti, S.: Data Mining for hypertext: a tutorial survey. ACM SIGKDD Explorations, Newsletter of the Special Interest Group on Knowledge Discovery and Data Mining (2000)

8. Papadakis, N.K., Skoutas, D., Raftopoulos, K., Varvarigou, T.A.: STAVIES: A System for Information Extraction from Unknown Web Data Sources through Automatic Web Wrapper Generation Using Clustering Techniques. IEEE Transactions on Knowledge and Data. Engineering 17(12), 1638-1652 (2005)

9. Liu, L., Buttler, D., Caverlee, J., Pu, C., Zhang, J.: A methodical approach to extracting interesting objects from dynamic web pages. Int. Journal of Web and Grid Services 1(2), 165-195 (2005)

10. Salton, G.: Introduction to Modern Information Retrieval. McGraw-Hill, New York (1983)

11. van Rijsbergen, C.J.: Information retrieval. Butterworths (1979)

12. Salton, G., Buckley, C.: Term Weighting Approaches in Automatic Text Retrieval. Technical Report TR87-881, Department of Computer Science, Cornell University, 1987. Information Processing and Management 32(4), 431-443 (1996)

13. Salton, G.: Automatic Text Processing. Addison-Wesley, Reading (1988)

14. Lee, D.L, Chuang, H., Seamons, K.: Document ranking and the vector-space model. Software, IEEE 14(2), 67-75 (1997)

15. Zhao, Y., Karypis, G.: Improving precategorized collection retrieval by using supervised term weighting schemes. In: Proceedings of the International Conference on Information Technology: Coding and Computing, 8-10 April 2002, pp. 16-21 (2002)

16. Martín del Brío, B., Sanz Molina, A.: Redes neuronales y sistemas borrosos Ra-Ma (2001)

17. Bouaziz, T., Wolski, A.: Applying Fuzzy Events to Approximate Reasoning in Active Databases. In: Proc. Sixth IEEE International Conference on Fuzzy Systems (FUZZIEEE'97), July 1-5, Barcelona, Spain (1997)

18. Ruiz, M.E., Srinivasan, P.: Automatic Text Categorization Using Neural Networks. Advances in Classification Research. In: Proceedings of the 8th ASIS SIG/CR Classification Research Workshop. Efthimiadis, E. (ed.) Information Today, Medford:New Jersey. vol. 8, pp 59-72 (1998) 